Recibido: 21-05-2014

Aceptado: 20-08-2014

Palabras clave: problemas específicos de aprendizaje, inclusión educativa, aula diversa, abordaje pedagógico, ritmos de aprendizaje.

Keywords: learning's specific problems, educational inclusion, diverse classroom, pedagogical approach, learning rhythms.

Patricia Medina Zuta

USIL pamezu_v@hotmail.com

\section{Los problemas específicos de aprendizaje: un reto de inclusión desde la diversidad del aula}

\section{Learning's specific problems: an inclusion challenge from diversity of classroom}

\section{Patricia Medina Zuta}

RESUMEN. Los problemas específicos de aprendizaje hanido evolucionando conceptualmente y demarcando nuevos escenarios en su afrontamiento dentro del aula. En este sentido, su tratamiento implica impulsar en el docente su apertura y compromiso respecto al reto de la inclusión desde el marco de un aula diversa.

El tratamiento de este tópico implica la reflexión sobre el abordaje pedagógico y la clarificación de ciertos procedimientos que comprendan el establecimiento de perfiles de inicio en el aula y el desarrollo de propuestas de intervención alternativa como la mediación entre pares; siendo el docente quien desde su rol facilitador demuestre su experticia en la gradualidad de los aprendizajes de acuerdo a las diferencias individuales y ritmos de aprendizaje.

\footnotetext{
ABSTRACT. Learning's specific problems have evolved conceptually and demarcated new scenarios in their coping within classroom. In this sense, their treatment involves promoting in teacher their beginning and commitment with regard to challenge of inclusion from framework of a diverse classroom.

The treatment of this topic involves the reflection on pedagogical approach and the clarification of certain procedures that include establishment of beginning's profiles in the classroom and the development of alternative intervention's proposals as peer mediation; being the teacher who since his role of facilitator demonstrates expertise in gradualness of learnings according to individual differences and learning rhythms.
} 


\section{Introducción}

D urante décadas, los problemas específicos de aprendizaje, se han manifestado como una prioridad en el ambiente del aula; primordialmente, en la Educación Básica Regular. Pero, ¿cuál ha sido el rigor de fijar en ellos una principal atención?, ¿qué pudo haber marcado un determinado tipo de intervención institucional, una vez detectados?, es acaso útil, seguir considerándolos "problemas", o es oportuno, empezar a mencionarlos como señal de una inevitable "diversidad".

En la actualidad, se utilizan una serie de conceptos que catalogan un problema de aprendizaje, algunos de ellos, referidos a los trastornos o desórdenes en uno o varios de los procesos psicológicos, que estarían relacionados con la comprensión del lenguaje en los niveles oral y escrito, y por consiguiente, con las habilidades que esto involucra, como el escuchar, hablar, leer, escribir, pensar, operar en el cálculo, entre los más representativos (Frith, 1989; Farnham-Diggory, 2004). Otra forma de denominar a los problemas de aprendizaje, se ha manifestado, al utilizar la palabra "retraso", y más específicamente, retraso en el desarrollo de un área en particular. Esto subrayaba en buena cuenta, una situación de “estándar homogenizante", al que todas las personas debieran responder.

Luego estarían, los comúnmente llamados "déficits", que según la Organización Mundial de la Salud (1992), implican toda pérdida o anormalidad de una estructura y/o función fisiológica, anatómica y/o psicológica. Pero ha sido mucho más contundente la atribución del concepto de discapacidad, toda vez, que este, pone en claro el impedimento de las personas para realizar alguna actividad de tipo conductual, comunicativa, de locomoción, entre otras, representativas del diario y cotidiano actuar humano (Verdugo, 1995). En una misma orientación de este concepto, el de minusvalía, suscribiría una innegable inclinación a deplorar la condición de una persona; señalando su desventaja frente a las demás, e incluso, acotando una mayor necesidad de dependencia (García, 1998).
Alétheia 2014, 2 (1) 21

Otro concepto muy común, fue el de excepcionalidad (Heward, 1998); que abarcaba a quienes sobrepasaban los límites de lo esperado en ciertos aprendizajes, lo cual fue relacionado con el término "talentoso" o "superdotado" (Galtón 1869 en Casanova 1999); y luego, la consideración de las necesidades educativas especiales (Warnock, 1987 en Romero y Lavigne, 2005), que no hacían, sino potenciar la situación de exclusión de las personas así identificadas, debido a sus diferencias en los distintos aspectos del desarrollo. Lo cual, más adelante, fue mucho más declarativo, cuando se les denominó en el rango de "capacidades diferentes".

Es interesante la orientación de Romero y Lavigne (2005), quienes consideran que las dificultades de aprendizaje, comprenden un grupo de trastornos que suelen confundirse unos con otros; y se atribuye esta permanente confusión a ciertos factores, no solo relacionados con la falta de claridad en su definición, sino con un probable "solapamiento" del término, el cual tiende a ser vinculado con determinadas razones de privación social y/o educativa, y con una realidad, en la cual, las diferencias, pueden ser motivo de una desigualdad en oportunidades. Ante tantas posturas conceptuales, no se puede sino identificar, un rasgo común a todas ellas, y esta termina siendo ilustrada, precisamente, en la presencia de una diversidad que impone la necesidad de nuevas miradas y formas de entender la realidad.

La educación especial ya no se define como la educación que reciben los alumnos y alumnas en razón del tipo de capacidad que presentan, sino como el conjunto de apoyos que el sistema pone al servicio de estos alumnos y alumnas para que puedan hacer efectivo su derecho irrenunciable a la educación (Coll, en Giné, 1995, s/p).

Si lo que se persigue es el desarrollo de los aprendizajes, y estos son en única medida, es probable que existan diferencias, que comprueben retrasos o adelantos frente a un estándar prefijado, lo que muchas veces se reconoce como "no alcanzar los logros esperados" (DCN, 2009), o quizás, sobrepasarlos. Pero, ¿cuáles son estos logros?; ¿siguen estando vigentes los estadios de desarrollo que planteara 


\section{UNIFÉ - EPG}

Piaget desde la década de los sesenta?, sobre todo, si analizamos, cómo las actuales y nuevas generaciones, presentan capacidades que traspasan las fronteras de la digitalización y de la inminente virtualización;así como el desarrollo de nuevas tendencias y habilidades en los sujetos de aprendizaje.

Si la escuela, sigue utilizando conceptos como "fracaso escolar", "repitencia", "bajo rendimiento", entre otros, se alude un sistema con indicadores encasillados a una única concepción y validación del desarrollo maduracional, prescrito en las llamadas "paralelas de normalidad" (Fortes del Valle, Ferrer y Gil, 1996). Sin embargo; esto no es nuevo, es parte del sistema, porque la organización del mismo, se ciñe a niveles y a grados de escolaridad; y no puede negarse la existencia de una estructura que ayude a organizar los períodos formativos. Es así como funciona en las distintas sociedades; siendo todo ello, producto de un conocimiento de muchos años de inversión en tiempo, procesos científicos, aportes teóricos, verdades absolutas e irrefutables.

Entonces, ¿se estaría considerando "intocable", el siquiera mencionar temas vinculados a promover nuevas consideraciones en el aprendizaje? Urge posibilitar "revoluciones científicas", que a saber de Kuhn (1972), son necesarias para seguir avanzando en lo que la realidad demanda. Entonces, se trata de aperturar paradigmas que promuevan la aceptación de la diversidad como un punto de partida, para proponer nuevas estructuras organizativas del avance escolar, y sobre todo, para reenfocar el desempeño docente. $\mathrm{Si}$ es claro que los modos, ritmos y estilos de aprendizaje no son los mismos ni están al mismo nivel, también es claro, que se requiere la presencia de pedagogos que puedan encajar en estas diferencias; siendo capaces de desempeñarse sin solo catalogarlas como dificultad o problema, o incluso derivarlas a otros especialistas, excluyéndose en la propia responsabilidad de afrontarlas.

El abordaje de los problemas de aprendizaje en el marco de la diversidad.

$\mathrm{Al}$ analizar los problemas de aprendizaje desde sus consideraciones más relevantes, quizás podamos ir encausando una forma mucho más accesible que integre la atención a la diversidad. $\mathrm{Al}$ respecto, se han hecho una serie de estimaciones sobre la prevalencia que pueden tener los problemas de aprendizaje en la última década; llegando a identificar entre un $8 \%$ y $15 \%$ de consideración de su presencia, como la principal causa de bajo rendimiento académico en la escuela (Jímenez, Guzmán, Rodríguez y Artiles, 2009). Incluso, estos mismos autores apuestan claramente, que los estudiantes que pudieran presentarlos, llegarían a evidenciar ciertos indicadores, tales como:

- Discrepancia: Señal comprobada de bajo rendimiento en más de un curso que esté relacionado al manejo de ciertos aprendizajes básicos.

- Exclusión: Se descarta las dificultades presentadas de orígenes sensoriales, emocionales, lingüísticos o de dispedagogía comprobada.

- Especificidad:Sedescartaalosestudiantescuyobajo rendimiento seageneralizado; independientemente de su edad o consideración estándar en la adquisición y consolidación de aprendizajes básicos, como el de la lectura, por ejemplo.

Sumado a ello, Romero y Lavigne (2005), proponen efectuar un diagnóstico, pero inciden principalmente, en que el mismo tenga un carácter de atención específica del trastorno, esto es, en principio definir el tipo de dificultad, y recién después, proponer la forma de detección, diagnóstico específico y el abordaje que se podría desplegar para una intervención conveniente. Sin embargo, no se descarta que gran parte de estas dificultades se produzcan por causas medioambientales y muchas veces sean detectadas debido a ellas; como es el caso por ejemplo, de la comprensión lectora, que llega a ser comprobable por la presencia de indicadores bastante identificables y comunes (dificultades de atención, problemas de memoria, entre otros). Habría que ver qué originó esta dificultad, qué factores la acrecentaron, y qué parte de la secuencia de desarrollo de esta función, no se llegó a completar (Giné, 2000).

El caso es, que tratándose de un proceso de aprendizaje, y entendiendo el mismo como un 
sistema general, que tiene su soporte en aprendizajes básicos afines a ciertas funciones mentales como la percepción, la memoria, la atención, entre las más relevantes; es pertinente asegurar el cumplimiento de una activación maduracional, como requisito de conformaciones cada vez más complejas en el aspecto funcional (Salgado, y Terán, 2008). Entonces, se podría constatar un punto central, sobre el cual se fija una estructura de base para el manejo de todo aprendiz, más allá de su estilo propio de aprender, o de las condiciones que le han significado un contexto de crecimiento y desarrollo. Desde este punto, se irán entretejiendo nuevas estructuras de capacidades cognitivas $y / o$ ejecutivas que le permitirán al estudiante poder seguir aprendiendo y ampliando sus estructuras mentales.

Hay que enfocar, que todo estudiante responde a factores intrínsecos y extrínsecos a su propio desarrollo, que pueden tener que ver con aspectos de naturaleza orgánica y/o experiencial, y que la versatilidad de una intervención ante una dificultad de aprendizaje, inicia justamente, en el establecimiento de ciertos procesos diagnósticos (Dueñas, 2002), que consideren el contexto al cual pertenece. En relación a ello, es pertinente la propuesta de Gil y Molero (2012, en Grau y Gil, 2012), acerca de efectuar un diagnóstico, que parte de formular una hipótesis de trabajo; llevando a cabo un protocolo de detección posible en su desarrollo, ejecutado por el propio docente y dentro del espacio del aula.

Esto le permitirá al docente, empezar a identificar los aciertos y desaciertos en términos de respuesta preestablecida para determinados aprendizajes, los aspectos más resaltantes de estas respuestas, sus relaciones, y sobre todo, lo que se hizo e intentó hacer para revertirlos. Es así como se reconoce una ruta de intervención secuencial:

- Análisis de la tarea: Conocimientos y estrategias que demanda, tiempo requerido y condiciones en general.

- Evaluación de la competencia curricular: Implica una observación directa, pruebas de rendimiento y entrevistas con los maestros.
- Evaluación psicopedagógica: Incluye etapas como la evaluación de tareas, de variables intervinientes, de procesos y de relaciones con pares y adultos.

- Evaluación de la familia: Va más allá del solo abordaje del sujeto, porque incluye su contexto de desarrollo y a los agentes familiares.

Mucho se ha asumido que las intervenciones diagnósticas lleguen a aplicarse cuando se observan problemas de respuesta a lo preconcebido como indicadores de desarrollo normal en los aprendizajes; y que si estos no responden a lo esperable, hay que poner en efecto todo un despliegue de acciones como las ya descritas; pero, qué podría obtenerse, si se somete todo ello a un abordaje del grupo en general; y se identifican las diferentes disposiciones y tipos de aprendizajes de cada uno de los estudiantes. Es probable, que este punto de partida diagnóstica, sea la acertada; evitando delimitar los casos a los que se atribuye la dificultad. Lo importante en este reenfoque, sería la disposición de no catalogar como bajo o alto a un aprendizaje; sino como "diferente". Otra vez, el reto es globalizar y entender la realidad de aprendizaje mediante un diagnóstico aplicable a todos, pero particularizante al desarrollo de cada quien.

$\mathrm{Si}$ el currículo prescribe una serie de competencias y capacidades como parte de un ciclo y así se conciben en el sistema, podría identificarse la disposición del aprendizaje real del estudiante, lo que ha podido conseguir hasta entonces, lo que está en proceso y tal vez, lo que no debería forzarse. En esta lógica, los maestros, responsables del progreso curricular en el aula, podrían estimular el desarrollo de aprendizajes de los estudiantes, a través de nuevos métodos incluyentes y accesibles a la diversidad, métodos, antes que clínicos, psicoeducativos (Grau y Gil, 2012; Giné, 2000).

El problema sustancial es conocer cuál ha de ser el papel que deben desempeñar los educadores y las escuelas en el próximo siglo. Para ello, es preciso hacer algunas reflexiones sobre la naturaleza de esas funciones y sobre las causas que las originan" (Santos Guerra, 2001, p. 152).

Es fundamental, quelos maestros comprendan las implicancias de la diversidad, lo que conllevará a 


\section{UNIFÉ - EPG}

que sepan asumir el reto de operacionalizar la teoría existente y convertirla en despliegues ejecutivos eficaces, a nivel de una pedagogía inclusiva en el aula. "Las limitaciones se ubican en el terreno de las experiencias en la práctica educativa" (Casanova y Rodríguez, 2009, p. 232) Urge desentrañar en cómo aplicar este enfoque; priorizando siempre las diferencias humanas (características evolutivas propias y ritmos de aprendizaje en interacción con las peculiaridades del contexto socio cultural en el que les toque desenvolverse). Entonces sería real, que se pueda brindar una respuesta educativa eficiente por su diversificación y adaptación al contexto (DCN, 2009). Existe una clara relación entre el comportamiento de los alumnos y lo que se espera de ellos. La baja expectativa hacia los alumnos con necesidades educativas especiales en el campo del aprendizaje o de la conducta social va a tener influencia en sus ritmos de aprendizaje (Marchesi y Martin, 1998, p. 29).

Atrás quedaron las pseudo civilizaciones que tenían bajas o nulas expectativas, en relación a aquellos niños que nacían diferentes por su discapacidad, y que presentaban algún signo de desventaja frente a los demás. Era común entonces, dejarlos morir o deshacerse de ellos; sobreponiendo objetivos sociales y políticos alineados con el poder o la supremacía frente a otras naciones, como fue el caso de la cultura espartana, que representó a una sociedad centrada en la guerra; buscando formar justamente eso, "guerreros"; y descartando a quienes no se ajustasen a este perfil (Ferraro, 2001). Históricamente, se mantuvo por mucho tiempo, un enfoque diferencial, en el que se propiciaba una reproducción de los sistemas políticos, y por ende un rol de la escuela, de corte academicista y técnico; validando procedimientos homogéneos; siendo impartidora de contenidos que eran dominados por los docentes; fomentando cada vez más, el rasgo social competitivo.

En este enfoque se alude a la desigualdad como reconocimiento de jerarquías según determinados criterios; propiciándose una lucha competitiva, que no hace más, que amenguar o anular, a quienes no se ajusten a la regularidad de estas exigencias, sobre todo si se trata de estudiantes con capacidades y ritmos de aprendizaje diferenciados, de quienes en más de una realidad, se tiene una baja expectativa. La amenaza se da, en tanto el régimen político, económico y social, subyacen a relevar un ordenamiento competitivo, que alinee al mundo educativo a una evaluación por resultados, más que de procesos individuales, y a la imposición de ciertas fuerzas de mercado, que superponen la sobrevivencia laboral y el cumplimiento obligatorio de un número de estándares durante todo el período formativo en las escuelas (Climént, 2010).

Un enfoque orientado a la diversidad promueve la socialización, la inclusión y el respeto por las diferencias, a través de un proceso de enseñanza y aprendizaje flexible, creativo y adecuado alas diferencias individuales de cada estudiante (DCN, 2009). Pero cómo lograrlo, si puede apreciarse la tendencia a convertir en "agreste" el período formativo, en tanto todo país aspira a alcanzar un estatus de desarrollo. La atención a la diversidad alude a la persona "tal y como esta es", sin cambiarla o cuestionarla por sus diferencias geográficas, de raza, sexo, ritmos de aprendizaje, etc. Entonces, se crea una necesidad y un foco de atención, que implica propiciar en todos los estudiantes, la toma de consciencia de la empatía, de la consideración por el otro, de asumir un rol que se oriente a conseguir una "convivencia pacífica", antes que un estatus de poder o de ventaja frente a otros.

Se trata de lograr en los estudiantes diferentes entre sí, procesos de construcción y deconstrucción de conocimientos a partir de su interacción, de la construcción de su identidad, y todo ello, porque la escuela sabe afrontar su rol en el desarrollo de estrategias y procedimientos educativos diversificados y flexibles (Jimenez y Vila, 1999). Pero esto exige un compromiso no solo de las autoridades y especialistas en temas curriculares, sino de quienes gestionan las instituciones educativas y de quienes imparten la enseñanza en los diferentes niveles educativos.

Los prejuicios que tienen hoy en día muchas escuelas y otras instituciones vinculadas al tema de "inclusión", casi siempre suelen relacionarse con un aparente temor ante aquello que no saben manejar; y esta amenaza, podría traducirse en considerar, el 
albergar a una población de alumnos con problemas de aprendizaje como una obligación impuesta por las leyes educativas; pero, qué tanto, esto sea un reflejo de su preocupación frente a las dificultades que les puedan generar ciertas postura de algunos padres de familia, o simplemente, su especial atención a lograr el cumplimiento en su totalidad, de los programas, antes que centrarse en lograr atender a la diversidad y sobre todo garantizar una igualdad de oportunidades en el sistema educativo (Bravo, 2010).

Es extraño comprobar que a pesar de la orientación del Diseño Curricular Nacional (2009), aún existan estos prejuicios por la falta de conocimiento; pero, es más aún contradictorio, constatar, que lo declarativo en temas de inclusión y diversificación curricular, muchas veces ni siquiera es comprobable en un modo procedimental que sea los suficientemente claro y apropiado al entendimiento tanto por parte de los propios especialistas, como por parte de quienes llegan a recibir las capacitaciones; es decir, los propios docentes que estarían en responsabilidad directa de la ejecución curricular. "...la institución educativa requiere identificar con claridad cuáles son las características de cada ciclo, y reconocer esa diversidad de estudiantes que tendrá en cada uno de ellos, para poder atender a todos respetando las diferencias" (DCN, 2009, p. 12).

$\mathrm{Si}$ el currículo, es diversificable, abierto y flexible como lo declara el DCN, esto debe ser comprobable en el momento en que puede ser contextualizado a la realidad del aula. Solo se estaría hablando de una verdadera adaptación curricular, si se comprobara que son los propios docentes quienes identifican la diversidad en el aula y saben cómo abordar las diferencias de aprendizaje existentes. El PEN al 2021, da cuenta de una sociedad cambiante y globalizada, que requiere que en temas de educación se brinde una enseñanza con propósitos claros; pero ¿qué tanto este afán es compatible con el primer paso que realmente implica una atención a la diversidad? No se puede ambicionar a tener todas las respuestas, y por lo mismo, todas las soluciones. Es más bien, acertado, poder ser estratégicos, ya que todo gran proyecto se logra paso a paso (Senge, 2000).
En primer lugar, toda institución educativa que realmente desea atender a la diversidad, necesita incorporar en su Proyecto Educativo Institucional este propósito y ello debe ser declarativo tanto en su identidad (Visión, Misión y Valores), como en su proyecto curricular. Los sistemas educativos deberían ser diseñados y los programas aplicados de modo que tengan en cuenta toda la gama de esas diferentes características y necesidades (UNESCO, 1990). Entonces se trataría de implementar una cultura organizacional en la que todos los agentes involucrados conozcan y manejen los conceptos de atención a la diversidad, más allá de los documentos declarativos o de los ideales que despliegan su importancia en la práctica institucional.

Parece una tarea sencilla; pero se requiere ser precisos en las orientaciones, de modo tal que no sea un discurso o una copia y pegado de lo que se plantea en diversos documentos; sino que efectivamente, la institución haga del proceso de enseñanza aprendizaje un proceso dinámico, en el cual se adapta el currículo a las reales necesidades de los estudiantes y a sus características y contextos socio culturales y liguísticos (DCN, 2009, p. 47).

El verdadero reto, entonces, implica saber operativizar esta atención a la diversidad; es decir, comprobar acciones concretas pero efectivas en este propósito (García, 2005). Ya en el marco de establecer una "una ruta de aprendizaje", los docentes podrían estructura su enseñanza; identificando claramente ciertos aprendizajes básicos que puedan adaptar a las necesidades e implicancias del aula; sin afectar el normal desenvolvimiento de su programa de estudios, esto es, "saber graduar los aprendizajes". Tampoco se trata de una aplicación ambiciosa en el cumplimiento de todos los procesos; pero en forma estratégica supone algunos pasos centrales como el desarrollo de un diagnóstico pedagógico (García, 2005), un abordaje que involucre a todos los estudiantes y una comprobación del avance o del cumplimiento de ciertos "mapas de progreso" que hoy se definen en las llamadas "Rutas de Aprendizaje" (MINEDU, 2013). 


\section{UNIFÉ - EPG}

Para esto, en el plano de una enseñanza por competencias, cuenta saber partir en coherencia con esta nueva mirada; es decir, tener claro desde el inicio qué aprendizaje se desea incorporar y que el mismo, le pueda ser útil al alumno y lo motive a seguir aprendiendo. Uno de ellos, podría el cálculo, otro la lectura, e incluso, en el plano conductual, la incorporación de ciertos hábitos. Lo central es decidir alguno de ellos para empezar; teniendo muy clara la competencia a lograr. Claro está, que esta competencia implicará, una serie de capacidades, destrezas y habilidades, que hay que desagregar como indicadores y criterios estructurados dentro de la gradualidad y de la propia pauta evaluativa.

$\mathrm{Al}$ respecto, es oportuna la mención de las rúbricas de evaluación como un sistema compatible con la verificación del avance según la posibilidad del estudiante (Simon, y Forgette-Giroux, 2001); esto se dará, teniendo en cuenta, la diferenciación de logros en los niveles de aprendizaje. Por ello, es relevante, que una rúbrica enfoque la capacidad y los criterios posibles a medir en las características específicas de este avance. Entonces puede tenerse en claro, qué es capaz de hacer el niño y qué se puede ir fortaleciendo o desarrollando; sin asignarle una etiqueta numérica que no detalle o caracterice su verdadero nivel de aprendizaje y lo que es capaz o no de hacer.

Es así, como la evaluación diagnóstica, va a representar una condición necesaria en el actuar pedagógico, ya que se convierte en un "diagnóstico pedagógico”, que a saber de García (2005, p. 88), es definido como "un tipo muy peculiar de evaluación que tiene en cuenta y se centra en las diferentes realidades que están implicadas en la enseñanza-aprendizaje de un alumno". Teniendo clara la competencia, capacidades, destrezas y habilidades a lograr, teniendo definido cómo y a través de qué instrumento evaluar, el docente puede hacerse cargo de todos los procesos a desarrollarse en el espacio del aula. Si se empieza por reconocer qué dificultades son las más comunes, o cuál podría ser una clasificación que facilite un entendimiento de las mismas, se estaría proponiendo un imput de actuación que alerte al docente en el inicio de la acción educativa. $\mathrm{Al}$ respecto, es conveniente la propuesta de Romero y Lavigne (2005), quienes plantean cinco grupos de reconocimiento de las dificultades de aprendizaje:

- Problemas escolares: Considerados problemas leves, que tienen su origen en factores externos al estudiante y que pueden ser resueltos a partir de la mediación educativa regularo del acompañamiento tutorial.

- Bajo rendimiento escolar: Considerados problemas de moderada gravedad y afectación personal, que tienen su origen en causas externas al estudiante, pero que combinados con ciertas características personales del estudiante pueden ser mucho más observables. Como se trata generalmente de dificultades a nivel de procesos psicolingüísticos, de motivación y/o de metacognicion; pueden ser recuperables a través de atenciones educativas escolares y familiares.

- Dificultadesespecíficasdeaprendizaje:Considerados problemas de moderadamente alta gravedad, que suelen tener su origen, independientemente de las condiciones medioambientales, pero se vinculan a factores educativos según su desarrollo y el grado de implicancia que puedan observar. Aquí tendría relevancia la necesidad de un rol de intervención especializada en paralelo a la propia consideración de la intervención del docente en el aula. De hecho, se trataría del establecimiento de una intervención coordinada entre docentes y especialistas, que involucren desde programas de intervención temprana, hasta adaptaciones curriculares individualizados al grado y/o características de la dificultad.

- Trastornos por déficit de atención e hiperactividad: Que en el rango de gravedad van a depender en su connotación, según el tipo e intensidad de las dificultades a nivel de respuestas que puedan estar teniendo los estudiantes en relación al desarrollo y ejecución de ciertas actividades en el aula. Se liga su origen a un factor netamente personal y son catalogados comúnmente, como parte de un síndrome. Al ser así, es comprobable el incremento y/o severidad que pueda tener el problema y cómo muchas veces, no solo necesita de una intervención 
psicoeducativa, sino también de tratamientos farmacológicos. Aunque, se ha podido apreciar que esto último, puede ocasionar ciertos efectos secundarios que desvían la dificultad a otro tipo de problemas; sin necesariamente encausar al alumno al objetivo de aprendizaje.

- Discapacidad intelectual Límite: Considerada una dificultad de causas personales graves porque estarían profundamente afectadas ciertas áreas dominantes; siendo esta una situación crónica. Si bien la estimulación ambiental puede ayudar a observar mejoras, no soluciona íntegramente el cuadro y el proceso de abordaje puede resultar bastante lento en el estudiante, en comparación con el desarrollo verificable en los demás compañeros.

Cada una de estas dificultades de aprendizaje, estaría representando un punto de partida para el establecimiento de una línea de base en el aula, y es el docente, quien destacando la orientación de aprendizajes básicos esperados, somete a diagnóstico las causas de la dificultad y busca cómo adaptar su intervención al ritmo de desarrollo de la clase, integrando a todos sus alumnos en el proceso. Claro está, que si la dificultad presenta características que la agravan, y requiere una complementaria terapia de intervención, la misma no debe obviarse en la intención de brindar al estudiante la posibilidad de desarrollarse mejor y de adquirir capacidades que lo incluyan en su desarrollo y en el proceso de aprendizaje de la clase (Arco y Fernández, 2004).

\section{A modo de conclusión.}

El docente puede lograr afrontar el reto de inclusión educativa, siendo abierto y flexible a metodologías que se propicien desde entrenamientos de ciertas habilidades, hasta ejecución de proyectos multidisciplinarios que induzcan la motivación, la ayuda mutua y la igualdad de oportunidades entre sus estudiantes. Todos se orientan a una misma actividad, pero van planteando sus roles y compartiendo espacios que les ayuden a desarrollarse mejor en los diferentes planos, no solo en el cognitivo.

\section{Referencias}

Alétheia 2014, 2 (1) 27

Arco, J. L., y Fernández, A. (2004). Manual de evaluación e intervención psicológica en necesidades educativas especiales. Madrid, España: McGraw-Hill.

Bravo, L. (2010). Prácticas inclusivas en el aula: Validación de un instrumento para conocer la perspectiva del alumnado de Primaria y Secundaria. Actualidades de Investigación en Educación, 10 (3), pp. 1-20. Universidad de Costa Rica. Recuperado de http:/ / revista.inie.ucr.ac.cr Casanova, N. (1999). La educación especial del niño excepcional. $\left(2^{\circ}\right.$ ed.). San Juan, Puerto Rico: Publicaciones puertorriqueñas.

Casanova, M.A. y Rodríguez, H. (2009). La inclusión educativa un borizonte de posibilidades. Madrid, España: La Muralla.

Climént, J. (2010) Evaluación de competencias en niños: Un error de apreciación y perspectiva. Actualidades de Investigación en Educación, 10 (3), pp. 1-25. Universidad de Costa Rica. Recuperado de http:/ / revista.inie.ucr.ac.cr

Consejo Nacional de Educación. Proyecto Educativo Nacional al 2021 - PEN (2006). La educación que queremos para el Perú. Lima, Perú. Recuperado de http://www.cne.gob.pe/docs/cne-pen/PENOficial.pdf

Ministerio de Educación del Perú (2009). Diseño Curricular Nacional de Educación Básica Regular. Recuperado de http://www.santillana.com.pe/ dcn_2009.pdf

Dueñas, M. L. (2002). Diagnóstico Pedagógico. Madrid, España: UNED

García, N. (1998). Manual de dificultades de aprendizaje. Lenguaje, lectoescritura y Matemáticas. Madrid, España: Narcea.

García, N. (2005). Marco de referencia actual para el diagnóstico pedagógico. Tendencias pedagógicas, 12 (83). Recuperado de

http:/ / www.tendenciaspedagogicas.com/ Articulos/2007_12_04.pdf.

Giné, C. (2000). Intervención psicopedagógica en los trastornos del desarrollo. Barcelona, España: EDIUOC.

Giné, C. (1995). Entrevista con César Coll sobre 
28 UNIFÉ - EPG

el nuevo real decreto de educación especial. Aula de Innovación Educativa. Revista Aula de Innovación Educativa, 45.

Recuperado de http://docs.grao.com/ artxml2pdf/entrevista-con-cesar-coll-sobre-elnuevo-real-decreto-de-educacion-especial $/ \mathrm{mm}$ d/1MDljOGMxNTMzNmY1NDFkZmJmZ DkyNDMwZTlmMWQtQVUtRVM=

Grau, C. y Gil, M.D. (2012).Intervención psicoeducativa en necesidades especificas de apoyo educativo. Madrid, España: Pearson Educación.

Farnham-Diggory, S. (2004). Dificultades de aprendizaje. (4ta ed.). Madrid, España: Ediciones Morata S.L. Recuperado de http://books.google.com. pe/books?id $=$ LXGrhVPfDAkC\&printsec $=$ fro ntcover\&hl $=\mathrm{es} \# \mathrm{v}=$ onepage\& $\& \& \mathrm{f}=$ false

Ferraro,P.(2001). Globalhabitatprotection:Limitations of development interventions and a role for conservation performancepayments. Conservation Biology, 15 (4). pp. 990-1000. Recuperado de http://www2.gsu.edu/ wwwcec/docs/ globalhabitatprotectioncb.pdf

Fortes del Valle, M.C., Ferrer, A.M., y Gil, M.D. (1996). Basespsicológicas de la educación especial: aspectos teóricos $y$ prácticos. Valencia, España: Promolibro.

Frith, U. (1989). Autism: Explaining the enigma. Oxford, Inglaterra: Blackwell.

Heward, W. (1998). Niños excepcionales: Una introducción a la educación especial. Madrid, España:Prentice-Hall. Jimenez, E., Guzmán, R., Rodríguez, C. y Artiles, C. (2009). Prevalencia de las dificultades específicas de aprendizaje. Revistas um.es. 25 (1).

Jimenez, F., y Vilá, M. (1999). De la educación especial a la educación en la diversidad. Málaga, España: Aljibe.

Kuhn, T. (1971). La estructura de las revoluciones cientificas. México D. F., México: Fondo de Cultura Económica.

Marchesi, A. y Martín, E. (1998). Del lenguaje del trastorno a las necesidades educativas especiales. Madrid, España: Alianza Editorial.

MinisteriodeEducaciónPerú(2013).Rutasdelaprendizaje. ¿Qué y cómo deben aprender nuestros niños y niñas? Fascículo 1. Comprensión de textos. 5 años de Inicial, Primery Segundo grado de Primaria. Recuperado de http://www2.minedu.gob.pe/umc/ece2011/ Rutas_de_aprendizaje/COMO_DEBEN_ APRENDER_NUESTROS_NINOS/ COMPRENSION_17-de-mayo[1].pdf

Organización Mundial de la Salud (1992).Clasificación Estadística Internacional de Enfermedades. Décima edición (CIE-10). Ginebra, Suiza: Organización Mundial de la Salud. Recuperado de http:// ais.paho.org/classifications/Chapters/pdf/ Volume3.pdf

Romero, J.F. y Lavigne, R. (2005). Dificultades en el aprendizaje. Vol. I. Definición, características y tipos. Junta de Andalucía, España: Consejería de Educación.

Salgado, A M. y Terán, N. (2008). Dificultades infantiles de aprendizaje. Detección y estrategias de ayuda. Madrid, España: Equipo Cultural.

Santos, M. A. (2001). Enseñar o el oficio de aprender. Organización escolar y desarrollo profesional. RosarioSanta Fe, España: Homo Sapiens Ediciones.

Secretaría del Foro Consultivo Internacional sobre educación para todos (1990). Declaración Mundial para todos. New York, Estados Unidos: UNESCO.

Senge, P. (2000). La danza del cambio. Bogotá, Colombia: Norma

Simon, M., y Forgette-Giroux, R., (2001). A rubric for scoring postsecondary academic skills. Practical Assessment, Research \& Evaluation, 7 (18). Recuperado de http://pareonline.net/getvn. asp? $\mathrm{v}=7 \& \mathrm{n}=18$

Verdugo, A. (1995). Personas con discapacidad. Perspectivas psicopedagógicas y rehabilitación. Madrid, España: Siglo XXI. 